\title{
Towards Energetic Autonomy of UAV
}

\author{
Krzysztof MATEJA ${ }^{1}$ and Wojciech SKARKA \\ Silesian University of Technology, Faculty of Mechanical Engineering, Poland
}

\begin{abstract}
This article presents the results of work of power supply system of an unmanned aerial vehicle (UAV) powered by solar cells. The UAV power supply system consists of solar cells, a charge controller, battery cells and a BMS (Battery Management System). During the designing process various options for energy acquisition and recovery was considered, in particular ATG (Advanced Thermoelectric Generator). The MBD (Model-Based Design) methodology was used to develop the UAV power supply system. The system was developed in simulation model and next it was studied to find the space of possible solutions using this model. Solar cells are the most efficient if the sun rays fall on them perpendicular. During the simulation various angles of inclination of solar cells in relation to sun rays were studied. These values depend on latitude, azimuth, season (length of day), weatheri.e. if there are any clouds and even air pollution. The power supply system had to be constructed in such a way to ensure during the day excess to energy enabling the operation of the engines, peripheral devices (sensors, measuring devices, GPS module) as well as charging the batteries to maximum capacity. The next step was related to the proper selection of battery cells to ensure the operation of the devices and flight at night. The whole research was additionally extended by minimizing the mass of power supply elements while increasing the ability to achieve energy autonomy. The developed system allows to increase the UAV flight duration, and with appropriate construction, geographical location and favorable weather conditions it is able to provide full energy autonomy of the UAV. The UAV powered by solar cells enables for example monitoring of pollution, boundaries, power lines, crops and measuring selected physical quantities over any area e.g. smog.
\end{abstract}

Keywords. UAV, Model-Based Design, Solar-powered aircraft, Energy harvesting, Energy autonomy

\section{Introduction}

Electric vehicles are gaining more and more popularity. Into this group we can also include unmanned aerial vehicles (UAV). These innovative vehicles are increasingly used in everyday life. UAVs are used, among others for distributing shipments [1], but also for more advanced operations, e.g. monitoring of borders or crops [2,3]. Currently, the biggest problem associated with UAVs is the increase of flight duration for longer operations without unnecessary landing. For this purpose, a system should be developed that will not only increase the flight duration, but also optimize the system in terms of weight. Therefore, an innovative approach to solving the problem is necessary. One should strive to obtain energy from the outside, but also strive to reduce energy consumption. By using the Model-Based Design (MBD) method [4], it will be possible

\footnotetext{
${ }^{1}$ Corresponding Author, Mail: krzysztof.mateja@polsl.pl.
} 
to analyze the power supply system in terms of energy demand, reduce the system weight to a minimum, and provide an energy surplus that will allow continuing operations at night $[2,5]$. The simulation should take into account various fields of technology that significantly affect the energy obtained by solar cells and energy demand. The results are influenced by, among others, flight mechanics (lift forces, drag) and aerodynamics for given conditions [6,7] i.e. flight altitude or speed. In addition to mechanics, electrotechnical issues related to the design of energy-saving systems, processing and storage of energy should also be taken into account. Each simulation model can be further optimized for other solutions. It can be scalable and detailed by changing technical parameters for any individual subsystems.

\section{Energy autonomy}

The main problem that currently occurs in the case of electric UAVs is the limited battery capacity which causes limited flight duration. There is a possibility of adding more battery cells but it will increase the weight of the vehicle. This is especially important for flying vehicles, where weight plays a very important role. The goal is to build the lightest aircraft possible. Energy storage sources are becoming more and more innovative, however, energy density remains the most important challenege for batteries. To increase flight duration and even ensure full energy autonomy, UAV aims to obtain energy from outside. One of the possibilities is obtaining solar energy through solar cells mounted on the aircraft structure. Another alternative is the use of the Advanced Thermoelectric Generator (ATG). ATG works on the principle of the Peltier phenomenon. The temperature difference between the two surfaces makes it possible to generate the electrical voltage needed to charge batteries or power electric drives. It is possible to use a hybrid system. One of the methods is to combine a solar power supply system with ATG [8].

To increase the efficiency of energy conversion, it is also possible to use supercapacitors. Their biggest advantage is short charging time, but also high efficiency and lifetime as well as high value of unit power $(\mathrm{W} / \mathrm{kg}$ - about 10 times higher than for lead-acid batteries). Due to the low internal resistance, it is possible to draw and release large currents in a short time. The disadvantage of supercapacitors is the low voltage of one element, however, the assembly of individual parts into serial, parallel or mixed modules is very simple. Supercapacitors can be connected together in the same way as individual battery cells [9].

The use of hydrogen fuel is another alternative to the UAV power supply system. The use of hydrogen as a fuel works well in vehicles where mass plays a greater role than volume, e.g. rockets, aircrafts. The downside ofthis solution is the inability to achieve full energy autonomy of the vehicle in a technically easy way. A hydrogen fueled vehicle is also certainly the most complicated to build, and the integration of individual modules requires the involvement of specialists from many industries in the project [10]. The alternative is also to combine the above methods with a certain percentage in one hybrid power system. The biggest disadvantage of this solution is the very high complexity of power supply system. Along with a more complex system, mass is also increasing. The complexity of the system can cause many problems related to UAV repair and maintenance [11].

UAV's power supply system can be build using many different power sources and storage sources (Fig.1). In the Fig.1 power sources and storage sources are gathered in 
one group as elements thanks to which electrical consumption devices are able to work. In our case study we want to develop power supply system for HALE (High-Altitude Long Endurance) which ultimately is to fly in the stratosphere. In HALE we can not use all power and storage sources. Some part of them will be not effective e.g. ATG. In the UAV temperature differences is most of the order of a few tens of degrees centigrade. This cause that this power source will not be efficient enough. Also supercapacitors will not be good choice as storage source for HALE. Supercapacitors can be use as storage source for FC(Fuel Cell) and only in the case of high dynamics of energy demand change but not for PV(Photovoltaics) powered facility with an even and slowly varying energy demand. For HALE the best options seems to be solar cells. In this article are the results of work on the power supply system for UAV which is powered by solar cells.



Figure 1. General scheme of UAV's power supply subsystems.

\section{Methods of analysis of energy-efficient systems}

The aim of energy efficiency analysis is to maximize flight duration and even ensure full energy autonomy. One of the methods to increase flight duration is to use solar cells. The solar cells power supply system consists of solar cells, charging regulator and battery cells. The only unknown value is the value of energy that can be obtained at a given time $\mathrm{t}[\mathrm{s}]$ from solar cells. The value of the power obtained by solar cells depends on several factors, among others:

- $\quad$ flight altitude

- angle of the sun towering over the horizon

- azimuth angle,

- seasons (duration of the day),

- cloud cover, fog

- air pollution

In the initial phase, it essential is to read the information that limits the access of sunlight, i.e. the angle of the sun towering over the horizon and the azimuth angle. Theinformation is directly related to the seasons, due to which the duration of day and 
night is constantly changing (the exception is the equator). Depending on the latitude the first angle of incidence of sunlight differs. This value is associated with the earth's circulation and is different every day. The second value is the azimuth angle, which changes all the time during the day. It depends on the rotation of the earth. The graph below shows how the solar cells performance changes for given angles of the sun towering over the horizon and azimuth angles(Fig. 2). The value is the resultant of sine angles of sun towering over the horizon and azimuth. We can use below equation to describe it as:

$$
\text { performance }=\sin \alpha \times \sin \beta
$$

Where:

$\sin \alpha-$ sine angle of the sun towering over the horizon $\sin \beta-$ sine angle of the azimuth

Cloudiness and air pollution are values that are studied by meteorological institutes. This data varies depending on location, season and weather conditions. The octane scale is used to determine cloud cover (from 0 - no cloud cover, to 8 - full cloud cover). In addition to the degree of sky coverage by clouds, the type and typess of clouds are also given. All this information has an impact on the value that we will finally get as input power to the power supply system $[12,13]$. The percentage values for the perpendicular angle of sunlight in the octane scale from 0 to 8 can be defined as: for 0 $100 \%, 1-98 \%, 2-94 \%, 3-88 \%, 4-79 \%, 5-70 \%, 6-54 \%, 7-50 \%, 8-27 \%$ [11].

The last variable that affects the value of the obtained power is air pollution. This value not only contains data concern on smog, but also dust layers that settle on solar cells. It is very important to determine the location of operations performed by UAVs. In the winter, soot accumulates on the solar cells, in addition we have to deal with dust. Also, moisture in the air can limit the value of obtained power. There may be frost or rime on the solar cells. Desert dust may occur in desert areas, which will also reduce the value of supplied power [14].

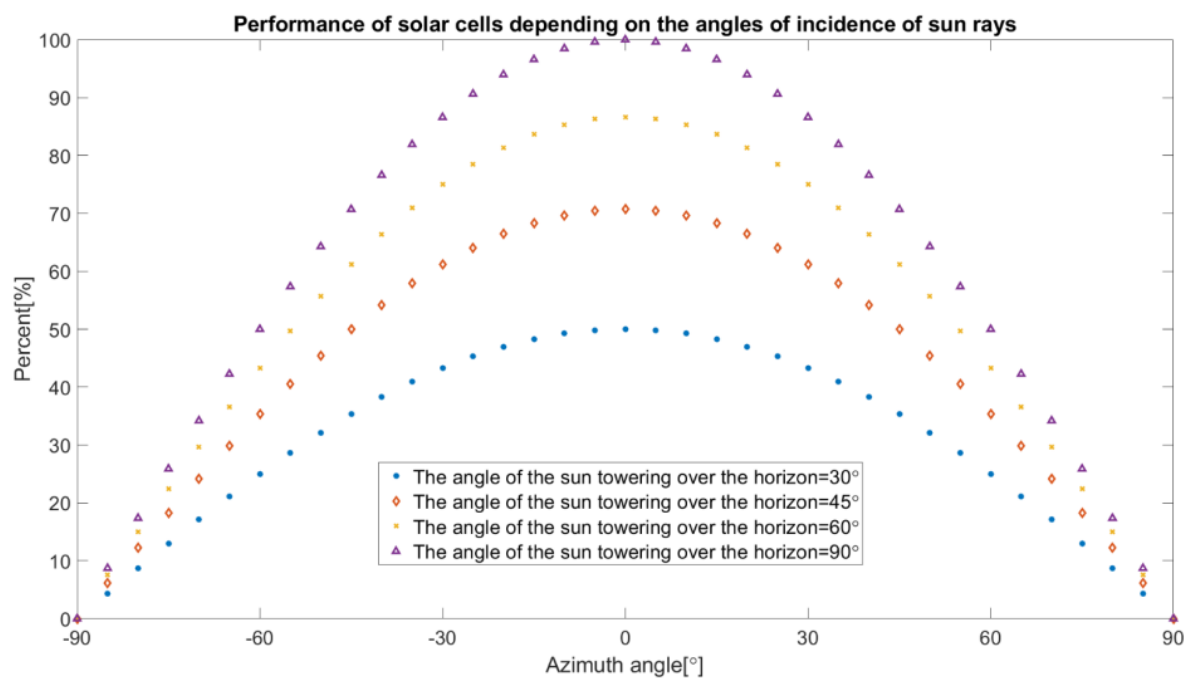

Figure 2. Graph of solar cells performance depending on the angle of incidence of sun rays. 


\section{Case study - energy-efficient UAV}

\subsection{Assumptions for energy-efficient $U A V$}

The object that was analyzed is UAV, which is to fly in the stratosphere (TWIN STRATOS). Its main source of power are solar cells (Fig. 3). Based on the construction data and flight parameters, the elements of the power supply system and the propulsion system were selected. The most important parameters of TWIN STRATOS:

- Mass: $70[\mathrm{~kg}]$

- Wingspan: $24800[\mathrm{~mm}]$

- Area of the wings: $34.6\left[\mathrm{~m}^{2}\right]$

- Area of tail-plane: $7\left[\mathrm{~m}^{2}\right]$

- Area of the solar cells: $19.7\left[\mathrm{~m}^{2}\right]$

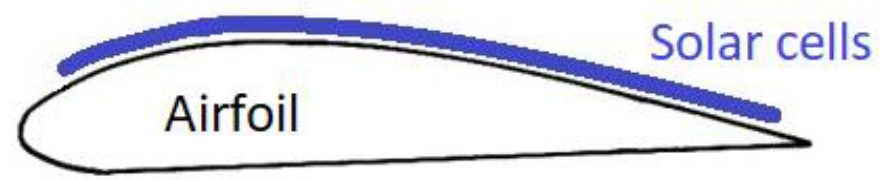

Figure 3. Solar cells attachment point on the Twin Stratos wing.

Solar cells and batteries are important elements of the system. Their selection is to ensure UAV full energy autonomy, with the lowest possible weight of the system. The power system is based on a voltage of 48 [V]. Initial parameters of the power supply system were determined on the basis of simplified analytical calculations. Thanks to them, the number of solar cells and batteries has been preselected. Another approximation is the analysis presented in the article. The battery capacity was initially determined as $72.5[\mathrm{Ah}]$ to provide the flight for $12 \mathrm{~h}$ at height $5500 \mathrm{~m}$ at night. A UAV flight simulation scenario was also developed (Fig. 4), which took into account the basic operations performed during the flight. The scenario helps in the of UAV energy demand so that it is possible to determine the power needed for take-off, altitude maintenance, climbing and landing.

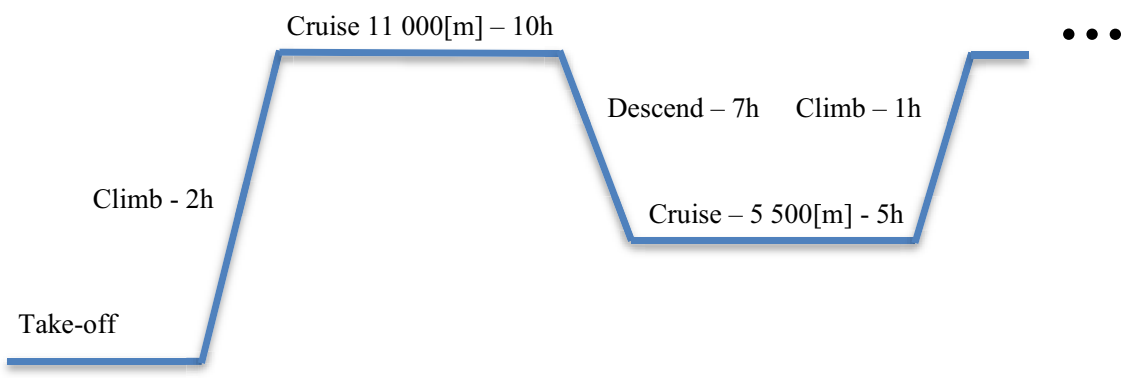

Figure 4. UAV flight scenario included in the simulation. 
The last issue is the selection of batteries to accumulate energy and ensure the operation of the system for the longest possible time. The technical parameters of the whole system should be consistent. The propulsion system, flight control system, communication and payload can be supplied with different voltages. For this reason, DC/DC converters must be used. The elements of the power supply system should take into account the lowest possible mass of the system and ensure safety and prevent short circuits and explosions of the battery cells.

\subsection{Concept of solar power supply system}

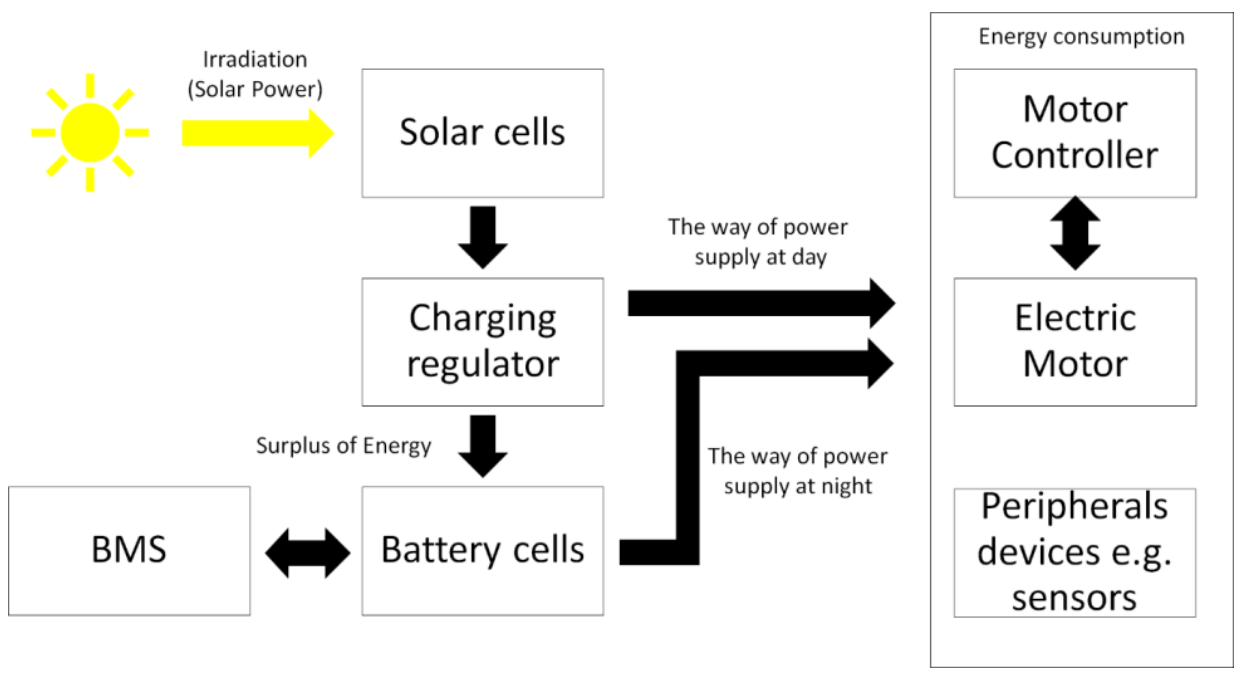

Figure 5. Schematic diagram of the power supply system.

The study assumed that the length of the day would be limited to 12 hours (to the equinoxes). Thanks to this initial thesis, it will be later possible to decide whether the received power is sufficient and the research can be directed to increase the length of the night or on the contrary, the day duration for operation should be extended. The area of Poland was adopted as the location for the study. Data regarding the angle of the sun over the horizon and azimuth angles were obtained from calculators [15]. Cloudiness values and value of air pollution, were omitted in the preliminary tests. We assumed to start UAV in sunny day. For our assumptions, i.e. the location adopted for Poland and the spring or autumn equinox, air pollution will not be as important as e.g. in the winter, when we deal with the biggest pollution e.g. smoke form chimneys. In this case air pollution has been omitted.

The power supply diagram (Fig. 5) helped developed a model for analysis. This is a special case of the HALE power supply system based on Fig.1. It is based on solar cells as power source and battery cells as storage source. The system simulation model was developed based on a single solar cell subsystem (Fig. 6). The cells are connected in series and in parallel, respectively, to ensure proper voltage and output current. The input value is the product of the sun irradiation $\left(\sim 1000 \mathrm{~W} / \mathrm{m}^{2}\right)$, the angle of incidence of sunlight relative to solar cells, cloud cover [\%] and air pollution [\%]. The analyzed 
HALE is beyond the influence of weather phenomena having a significant impact on solar radiation. In this case and approximation cloud cover has been omitted. Significant impact on this type of phenomena occurs in the case of LALE(Low-Altitude LongEndurance).

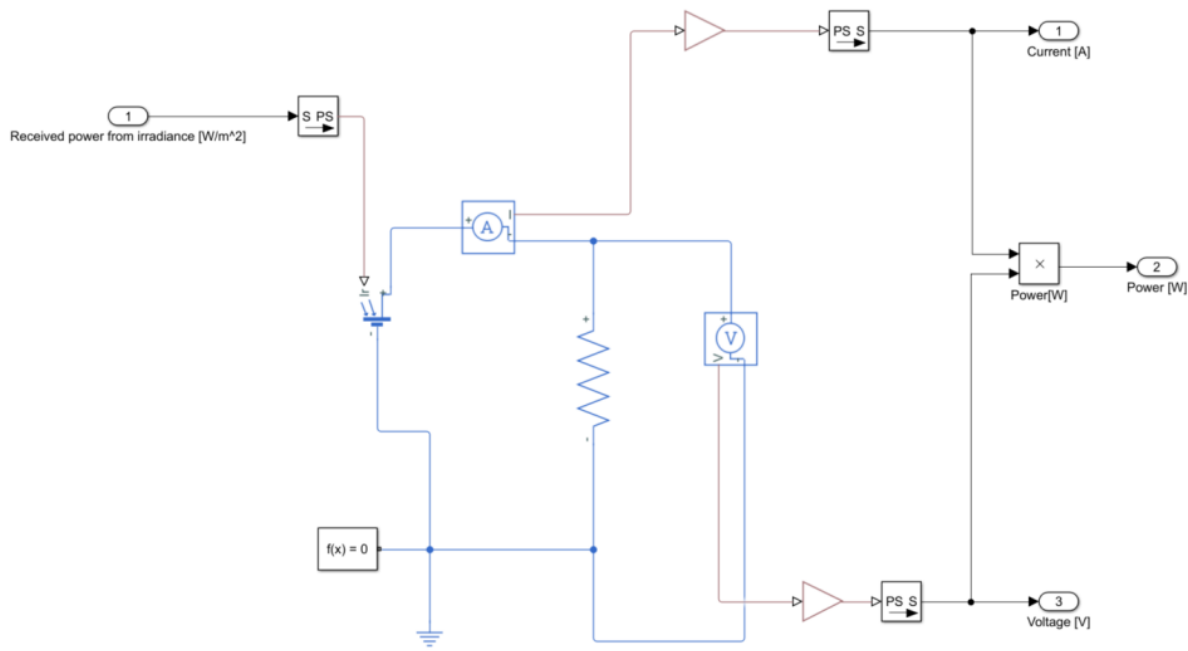

Figure 6. Single solar cell model.

In addition to the solar cell model, a battery discharge and charging model was also developed (Fig. 7). This model is to determine the energy balance and check properly connected batteries in modules which are able to provide flight maintenance for UAV at night $(\mathrm{t}=12 \mathrm{~h})$.

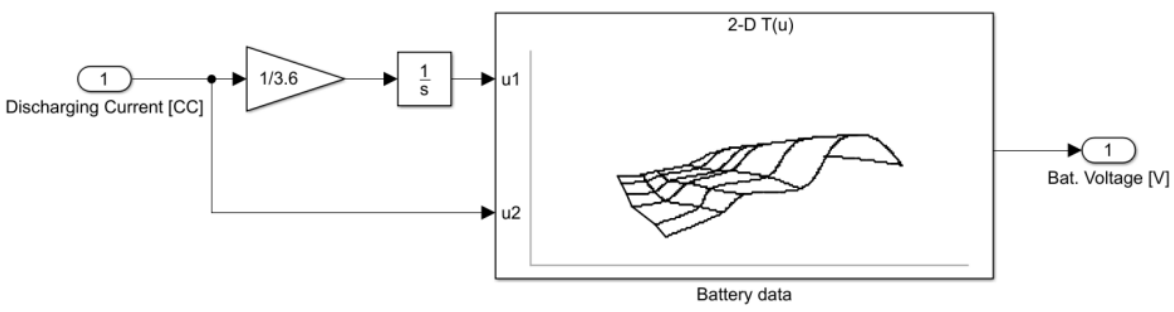

Figure 7. Model of charging and discharging the battery.

The Battery data block contains the values of discharge currents, capacitance and voltage of Li-Ion cells. Thanks to this data, it is possible to perform a simulation that illustrates how the battery will behave after loading with the set current value I [A].

The single models were combined into subsystems and then integrated into one system, enabling the simulation of energy balance for UAV powered by solar cells. 


\subsection{Analysis of energy consumption in various operational states}

Energy demand data for various TWIN STRATOS flight scenarios was developed by aviation constructors and designers. The extract from this data let calculate the energy consumption for the main flight phases (Table 1). In simulation model we used this values as constant to check the duration of flight and discharging time of the batteries.

Table 1. Energy consumption for main phases of flight for the UAV model.

\begin{tabular}{lc}
\hline Flight phase & Energy demand \\
\hline Take-off and climb V $=90 \mathrm{~km} / \mathrm{h}$ & $1100[\mathrm{~W}]$ \\
\hline Cruise speed $\mathrm{V}=55 \mathrm{~km} / \mathrm{h} h=11 \mathrm{~km}$ & $500[\mathrm{~W}]$ \\
\hline Average power needed to maintain flight at night & $250[\mathrm{~W}]$ \\
$\mathrm{V}=24 \mathrm{~km} / \mathrm{h}, \mathrm{h}=5.5 \mathrm{~km}$ & \\
\hline
\end{tabular}

Initial analysis of the maximum energy consumption is about $1200 \mathrm{~W}$, including the operation of additional devices (camera, GPS module, servos). The solar cells are able to provide a maximum of about $2500 \mathrm{~W}$. With this information, we are able to determine what voltage and current are able to "flow" to recharge the batteries. The data obtained in the simulation allow to state that the energy surplus during the day will easily charge the batteries to their maximum capacity during 12 hours of sunlight (Fig. 8). The guideline was also to take into account the margin of energy surplus for decisions in the event of a breakdown or other technical faults. This time has been initially set to 4 hours. The below chart shows linear approximation of the power consumption and power demand. We assumed that the UAV would take off with a discharged battery to check if the surplus energy from solar cells would be able to charge the batteries during the day. To prepare more detailed chart we have to use equation related to movement of the sun for a given localization, taking into account also movement of the UAV.

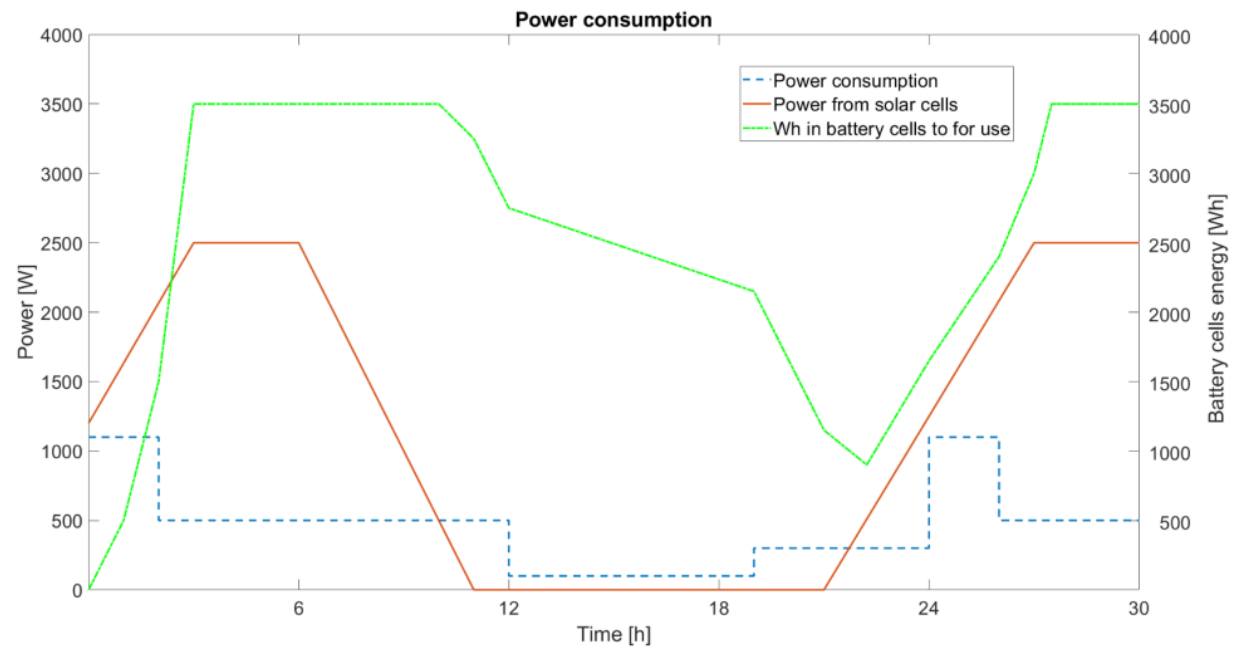

Figure 8. Energy demand graph for the example of TWIN STRATOS mission (from Fig.2). 
Battery discharge analysis was based on three scenarios:

- Scenario 1 - maintaining flight at night at an altitude of 11,000 [m]

- Scenario 2 - gliding from 11,000 [m] to 1,000 [m], next climbing to $11,000[\mathrm{~m}]$

- Scenario 3 - gliding from $11,000[\mathrm{~m}]$ to $5,500[\mathrm{~m}]$ and then maintaining the flight at this altitude (Fig. 4)

The analysis of battery discharges at night shows that the power needed to maintain the flight at night discharges the batteries after a maximum of about 19.5 hours. This analysis included gliding of UAV for 7 hours (scenario 3). The result is minimal power consumption and a minimum sink rate $(\sim 0.2 \mathrm{~m} / \mathrm{s})$. Gliding allows to use potential energy, so that for some time electric motors do not need to draw electricity from batteries (Fig. 9).

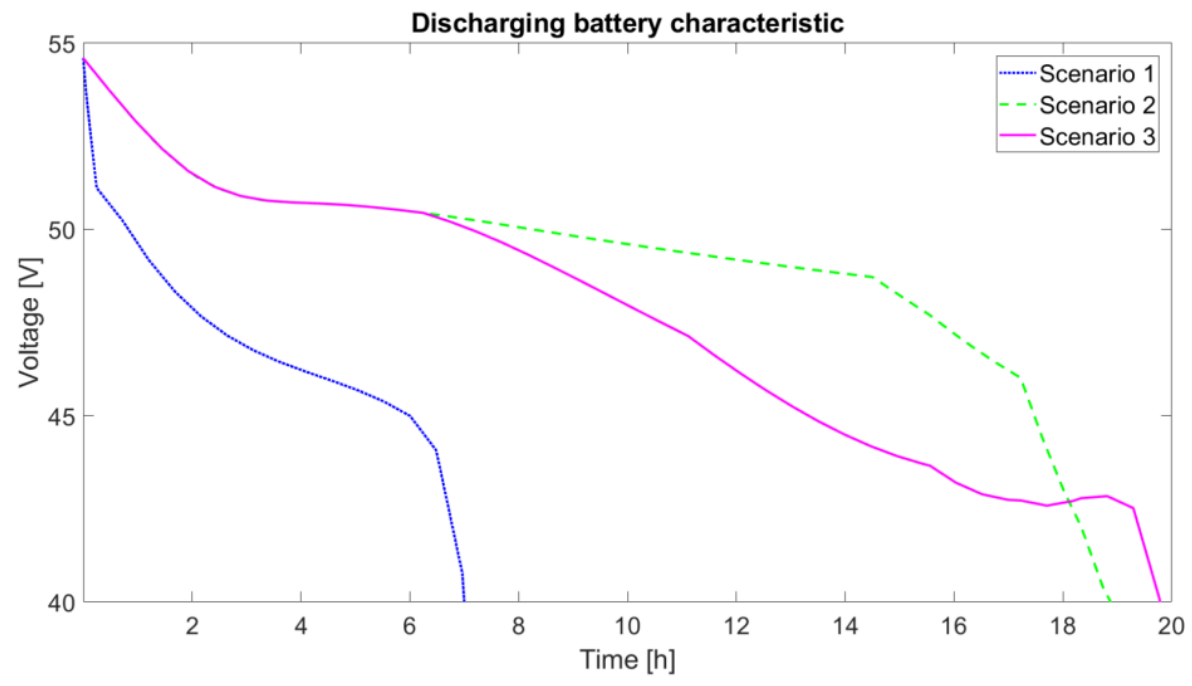

Figure 9. Battery discharge characteristics for different scenarios.

The comparision of scenarios 2 and 3 shows that the batery discharge time is similar. The most energy consuming is scenario 1. This analysis shows that except for the the energy stored in batteries it is also worth to use potential energy as time buffer. This will help to increase the duration of the flight.

\section{Discussion of results and conclusions}

Designing a solar powered UAV system is not only the field of electrical engineering, but also aviation mechanics, meteorology, astronomy and environmental protection. We should strive to develop a system that will satisfy and be able to ensure full UAV energy autonomy. Analysis of Fig.8 shows that the number of solar cells used in the simulation is sufficient. For prepared scenarios surplus of energy is significant and is not used in full. We can decide on two ways of action. First is connected with limiting the number of solar cells. Second is to increase the capacity of batteries. First way will allow to 
decrease the mass of the power supply system but also can cause shorter ranges in adverse weather conditions. Increasing the mass of batteries increase the range of UAV but mass is on of the most critical values so probably this way will be suitable only for relevant restrictions. Additionally we can reduce the batteries due to the use of potential energy. Developed simulations show that gliding can increase time of flight relative to maintaining the flight at least twice. It all depends on the starting ceiling. Before taking the road we have to prepare more detailed simulation model for specific flight scenarios and for specific latitude and flight conditions. Initial assessment is a somewhat hypothetical condition. A more detailed model will allow to specify critical locations, e.g. polar zones. Methods will be sought for modeling a hybrid UAV power system system and also for reducing the system's complexity.

\section{References}

[1] J. Doskocz, P. Kardasz, M. Hejduk, P. Wiejkut, The possibilities of using drones in the courier services, World Scientific News, Vol. 48, 2016, pp. 119-132. Available: http://psjd.icm.edu.pl/psjd/element/bwmeta1.element.psjd-d681cbe3-29e6-42c4-898b4132493a08ed/c/WSN_48_2016_119-132.pdf

[2] W. Skarka, Model-based design and optimization of electric vehicles, Advances in Transdisciplinary Engineering, 2018, Vol. 7, pp. 566-575.

[3] J. Blazakis, Border Security and Unmanned Aerial Vehicles, Connections, Vol. 5, No. 2, pp.154159. [Online]. Available: https://www.jstor.org/stable/10.2307/26323244

[4] W. Skarka, Methodology for the optimization of an energy efficient electric vehicle. In: Proceedings of the 6th International Conference on Integrity-Reliability-Failure, Lisbon, 2018, pp. 415-422.

[5] M. Targosz, W. Skarka, P. Przystałka, Model-Based Optimization of Velocity Strategy for Lightweight Electric Racing Cars, Journal of Advanced Transportation, Vol. 2018, Article ID 3614025, 20 pages. [Online]. Available: https://doi.org/10.1155/2018/3614025

[6] M. Wąsik and W. Skarka, Aerodynamic Features Optimization of Front Wheels Surroundings for Energy Efficient Car, Advances in Transdisciplinary Engineering, 2016, Vol. 4, pp. 483-492.

[7] M. Wąsik and W.Skarka, Design Optimization of Electric Propulsion of Flying Exploratory Autonomous Robot, Advances in Transdisciplinary Engineering, 2015, Vol. 2, pp. 367-376.

[8] P. Sundarraj, Theoretical and experimental analyses of advanced hybrid solar thermoelectric generator, Shiv Nadar University, Department of Mechanical Engineering, Gautam Buddha Nagar, 2017.

[9] Z. Juda, Supercapacitors applied to the energy recovery system in an electric drive vehicle, Technical Transactions. Mechanics, Vol. 6, 2008, pp. 191-199. Available: https://repozytorium.biblos.pk.edu.pl/resources/34108

[10] M. Tyczka and W. Skarka, Optimisation of Operational Parameters Based on Simulation Numerical Model of Hydrogen Fuel Cell Stack Used for Electric Car Drive, Advances in Transdisciplinary Engineering, 2016, Vol. 4, pp. 622-631.

[11] D. Rand and R.M. Dell, Hydrogen Energy - Challenges and Prospects. RSC Publishing, Cambridge, 2008.

[12] D. Matuszko, Influence of the extent and genera of cloud cover on solar radiation intensity, International Journal of Climatology, Vol. 32, 2012, pp. 2403-2414. [Online]. Available: https://rmets.onlinelibrary.wiley.com/doi/epdf/10.1002/joc.2432

[13] D.R. Myers, Solar Radiation: Practical Modeling for Renewable Energy Applications, CRC Press, Boca Raton, 2013.

[14] M.H. Bergin, C. Ghoroi, D. Dixit, J.J. Schauer and D.T. Shindell, Large Reductions in Solar Energy Production Due to Dust and Particulate Air Pollution, Environmental Science \& Technology Letters, Vol. 4, 2017, pp. 339-334. Available: https://pubs.acs.org/doi/10.1021/acs.estlett.7b00197.

[15] Sun calculator. [Online]. Available: www.suncalc.org 